\title{
rs621554 single nucleotide polymorphism of DLC1 is associated with breast cancer susceptibility and prognosis
}

\author{
$\mathrm{XIA} \mathrm{DING}^{1}$, SUMEI GAO $^{2}$ and QIFENG YANG ${ }^{2,3}$ \\ Departments of ${ }^{1}$ Oncology, ${ }^{2}$ Breast Surgery and ${ }^{3}$ Pathology Tissue Bank, \\ Qilu Hospital, Shandong University, Jinan, Shandong 250012, P.R. China
}

Received February 12,2016; Accepted March 7, 2016

DOI: $10.3892 / \mathrm{mmr} .2016 .4987$

\begin{abstract}
Deleted in liver cancer 1 (DLC1) on chromosome 8 p22, is an important tumor suppressor gene originally identified to be deleted in hepatocellular carcinoma. It can regulate the structure of the actin cytoskeleton and inhibit cell proliferation, motility and angiogenesis, which predominantly depends on its homology to rat RhoGAP. There are many genetic variants in DLC1, which may influence its antitumor efficacy. The rs621554 (IVS19+108C >T) polymorphism is a synonymous single nucleotide polymorphism (SNP) previously found to be associated with hepatocellular carcinoma. In the present study, 453 patients with breast cancer and 330 healthy females were analyzed using a cycling probe method. It was determined that the rs621554 polymorphism of DLC1 was associated with breast cancer susceptibility, with the CC and CT genotypes resulting in a higher risk of developing breast cancer. In regard to clinicopathological variables, it was demonstrated that the CT and CC genotype were associated with tumor size, lymph node metastasis and progesterone receptor status. Patients with the CT and CC genotype had shorter disease-free survival and overall survival rates compared with those with the TT genotype. Additionally, it was demonstrated that the rs621554 polymorphism was correlated with DLC1 expression at the mRNA level. These results suggested that the rs621554 polymorphism is associated with breast cancer susceptibility and prognosis, and may serve as a biomarker for breast cancer development and progression.
\end{abstract}

Correspondence to: Dr Qifeng Yang, Department of Breast Surgery, Qilu Hospital, Shandong University, 107 Wenhua Xi Road, Jinan, Shandong 250012, P.R. China

E-mail: qifengy_sdu@163.com

Abbreviations: DLC1, deleted in liver cancer 1; SNPs, single nucleotide polymorphisms; HWE, Hardy-Weinburg equilibrium

Key words: DLC1, breast cancer, susceptibility, prognosis, polymorphism

\section{Introduction}

Breast cancer is one of the most common types of malignancies among females worldwide and has become a serious health burden for women with 1,380,000 new cases and 458,000 breast cancer-related fatalities annually $(1,2)$. However, mechanisms underlying the onset and development of breast cancer remain to be fully elucidated. Like other malignancies, breast cancer development is the result of a combination of genetic and environmental factors (3-6). The most frequent type of genetic alteration, single nucleotide polymorphisms (SNPs), are known to be important carcinogenesis and cancer development $(7,8)$. It is well known that breast cancer is associated with SNPs in a number of genes, such as BCL-2 and XRCC3 $(9,10)$.

Deleted in liver cancer 1 (DLC1), mapped to chromosome 8 p22, is an important tumor suppressor gene originally identified to be deleted in hepatocellular carcinoma (HCC). Recently, increased attention has been paid to its actions in other types of malignancies, including breast, prostate, cervical and lung (11) cancer. DLC1 can regulate the structure of the actin cytoskeleton and inhibit cell proliferation, migration, invasion and angiogenesis, which predominantly depends on its homology to rat RhoGAP, which acts as a catalyzing enzyme in the conversion of the active GTP-bound rho complex to the inactive GDP-bound form (12-14). Mutation screening of DLC1 was performed in 159 patients with prostate cancer indicated 8 exonic and 23 intronic SNPs of DLC1; however, no association with cancer was identified (15). Among these mutations, rs621554 (IVS19+108C>T) polymorphisms, a synonymous SNP located 108 bp downstream of exon 19, was found to be correlated with HCC susceptibility in patients with hepatitis $\mathrm{B}$ virus (16). However, little is known regarding the influence of the DLC1 rs621554 polymorphism in breast cancer. Thus, the present study aimed to determine the association between the rs621554 polymorphism and breast cancer susceptibility, clinicopathological variables and prognosis.

\section{Materials and methods}

Patients and samples. This study involved 453 patients who were diagnosed with breast cancer and 330 healthy females who were recruited from annual physical examinations between 2008 and 2012 at the Qilu hospital of Shandong 

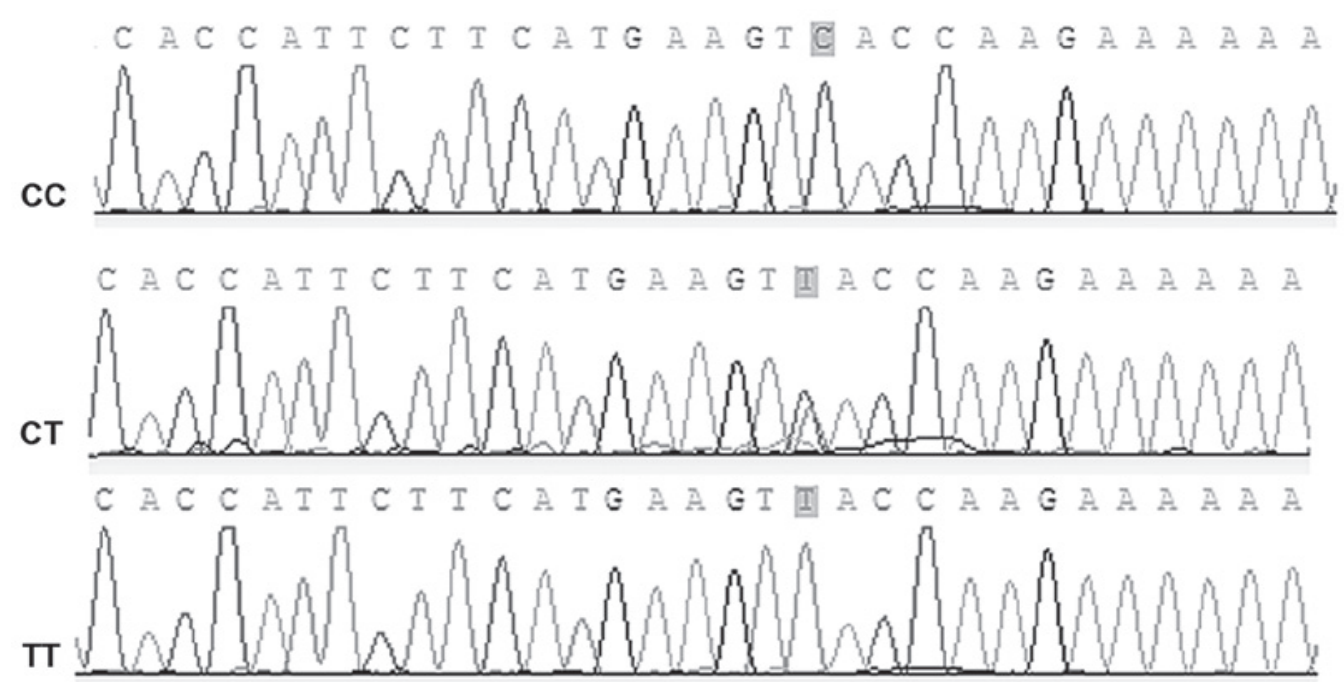

Figure 1. Genotyping results of rs621554 polymorphism were shown to be consistent using DNA sequencing and cycling probe methods. Genotype shown on the left indicated the results of the cycling probe and those highlighted in the figure show the results of the DNA sequencing based on different peaks.

University (Jinan, China). The average age of the case and control group was $50.00 \pm 11.40$ and $48.23 \pm 7.93$ years, respectively, and there was significant difference in the age of the two groups as determined by Student's t-test $(\mathrm{P}=0.367)$. In order to investigate the correlation between the DLC1 rs621554 polymorphism and changes in DLC1 mRNA levels, 20 tumor tissue samples were obtained from the patient group during tumor resection. To detect whether the rs621554 polymorphism is associated with breast cancer prognosis, 245 patients with long-term clinical follow-up were enrolled. The patients were followed up every three months, and disease free survival and overall survival were analyzed. Whole blood samples (200 $\mu \mathrm{l})$ were obtained from each individual in the case and control groups and were stored at $-80^{\circ} \mathrm{C}$ until use. The following clinicopathological characteristics were determined from patient notes: Age at diagnosis, pathological type, tumor size, histological grade, lymph node status, as well as biological markers, such as the presence of estrogen receptor (ER), progesterone receptor (PR) and human epidermal growth factor receptor 2 (HER2). This study was approved by ethical committee of Shandong University. Written informed consent was signed by each subject involved in the study.

DNA extraction. DNA was extracted from whole blood cells using the TIANamp Genomic DNA kit (Tiangen, Beijing, China) according to the manufacturer's instructions. DNA concentration and purity were measured using an ultraviolet spectrophotometer (GE Healthcare, Pittsburgh, PA, USA). The extracted DNA samples were routinely stored at $-20^{\circ} \mathrm{C}$.

SNP genotyping analysis. Cycling probes were synthesized by Takara Biotechnologies (Dalian), Co. Ltd. (Dalian, China), and an allelic discrimination assay [Cycleave PCR Core kit, Takara Biotechnologies (Dalian), Co. Ltd] was performed using an ABI 7900HT thermal cycler. The PCR amplification conditions were conducted according to the manufacturer's instructions: $95^{\circ} \mathrm{C}$ for $30 \mathrm{sec}, 45$ cycles of $95^{\circ} \mathrm{C}$ for $5 \mathrm{sec}$, $55^{\circ} \mathrm{C}$ for $10 \mathrm{sec}$, and $72^{\circ} \mathrm{C}$ for $25 \mathrm{sec}$. Several DNA samples were randomly selected for DNA sequencing to confirm the genotyping.

Reverse transcription-quantitative polymerase chain reaction analysis $(R T-q P C R)$. Total RNA was extracted using TRIzol reagent [Takara Biotechnologies (Dalian), Co. Ltd.]. The cDNA was reverse transcribed from $1 \mu \mathrm{g}$ total RNA using the PrimerScript RT Reagent kit [Takara Biotechnologies (Dalian), Co. Ltd.]. mRNA quantification was performed using SYBR PCR mix [Takara Biotechnologies (Dalian), Co. Ltd.] on an ABI 7900HT platform (Applied Biosystems, Foster City, CA, USA). Primer sequences were as follows: Forward: 5'-GGGCTGCTTTTAACTCTGGTAAAG-3' and reverse: 5'-CCATGGGTGGAATCATATTGG-3' for glyceraldehyde 3-phosphate dehydrogenase; and forward: 5'-CCAAACCCAAGACTACGGCTA-3' and reverse: 5'-GCTGTGCATACTGGGGGAA-3' for DLC1. Primers were obtained from Sangon Biological Engineering, Co., Ltd. (Shanghai, China). A 20- $\mu 1$ reaction system was used and contained $1 \mathrm{X}$ Cycleave PCR reaction mixture, $0.4 \mu \mathrm{M}$ cycling probe, $0.2 \mu \mathrm{M}$ PCR forward primer, $0.2 \mu \mathrm{M}$ PCR reverse primer and 50 ng DNA template. The cycling probe method was used, the CC genotype presented strong fluorescence, TT genotype presented no fluorescence and CT genotype present weak fluorescence (17). Sequence detection system software 1.2.1 (SDS1.2.1, Applied Biosystems) was used for analysis. The experiment was repeated in triplicate to confirm the results.

Statistical analysis. SPSS 18.0 software (SPSS Inc., Chicago, IL, USA) was used to analyze data in this study. The genotype and allele frequencies were tested using the public statistical web-tool http://www.oege.org/software/hwe-mr-calc.shtml for Hardy-Weinburg equilibrium (HWE). P $>0.05$ was considered to indicate non-deviation from HWE. Logistic regression models were used to analyze the association between the rs621554 polymorphism and breast cancer susceptibility, as well as the clinicopathological characteristics. Differences in patient survival rates were analyzed using the Kaplan-Meier 
Table I. Genotype distribution in cancer cases and controls.

\begin{tabular}{|c|c|c|c|c|}
\hline \multirow[b]{2}{*}{ Genotype } & \multicolumn{2}{|c|}{ No. of patients } & \multirow[b]{2}{*}{ P-value } & \multirow[b]{2}{*}{ OR $(95 \% \mathrm{CI})$} \\
\hline & Control (n=330) & Cancer $(n=453)$ & & \\
\hline TT & 54 & 44 & & \\
\hline $\mathrm{CT}$ & 166 & 220 & 0.032 & $1.627(1.041,2.541)$ \\
\hline $\mathrm{CC}$ & 110 & 189 & 0.001 & $2.109(1.328,3.348)$ \\
\hline
\end{tabular}

OR, odds ratio; $\mathrm{CI}$, confidence interval.

Table II. Association between rs621554 and clinicopathological variables.

\begin{tabular}{|c|c|c|c|}
\hline \multirow[b]{2}{*}{ Variable } & \multicolumn{2}{|c|}{ Genotype } & \multirow[b]{2}{*}{ P-value } \\
\hline & $\mathrm{TT}$ & $\mathrm{CC}+\mathrm{CT}$ & \\
\hline Age (years) & & & 0.561 \\
\hline$\leq 45$ & 20 & 148 & \\
\hline$>45$ & 24 & 214 & \\
\hline Pathological type & & & 0.759 \\
\hline Ductal & 37 & 296 & \\
\hline Other & 7 & 64 & \\
\hline Tumor size (cm) & & & 0.035 \\
\hline$\leq 2$ & 24 & 136 & \\
\hline$>2$ & 15 & 175 & \\
\hline Histological grade & & & 0.242 \\
\hline Low & 23 & 165 & \\
\hline High & 6 & 75 & \\
\hline Lymph node metastasis & & & 0.047 \\
\hline No & 28 & 170 & \\
\hline Yes & 14 & 167 & \\
\hline ER & & & 0.359 \\
\hline Negative & 26 & 178 & \\
\hline Positive & 16 & 149 & \\
\hline PR & & & 0.031 \\
\hline Negative & 12 & 151 & \\
\hline Positive & 30 & 176 & \\
\hline HER2 & & & 0.476 \\
\hline Negative & 38 & 283 & \\
\hline Positive & 4 & 44 & \\
\hline
\end{tabular}

ER, estrogen receptor; PR, progesterone receptor; HER2, human epidermal growth factor receptor 2.

method. The Cox hazard regression model was used to analyze the independent prognostic value of the rs621554 polymorphism. $\mathrm{P}<0.05$ was considered to indicate a statistically significantly difference.

\section{Results}

DNA sequencing shows complete reliability of genotyping using cycling probe method. Ten DNA samples randomly selected from the patient group were sent to Sangon Biological Engineering Co., Ltd. for DNA sequencing. As shown in Fig. 1, DNA sequencing showed consistent results, which suggest reliability of the genotyping method.

rs621554 polymorphisms are associated with breast cancer susceptibility. In total, 453 patients with breast cancer and 330 healthy females were involved in this study. Subjects from the two groups were confirmed to meet HWE using the $\chi^{2}$ 

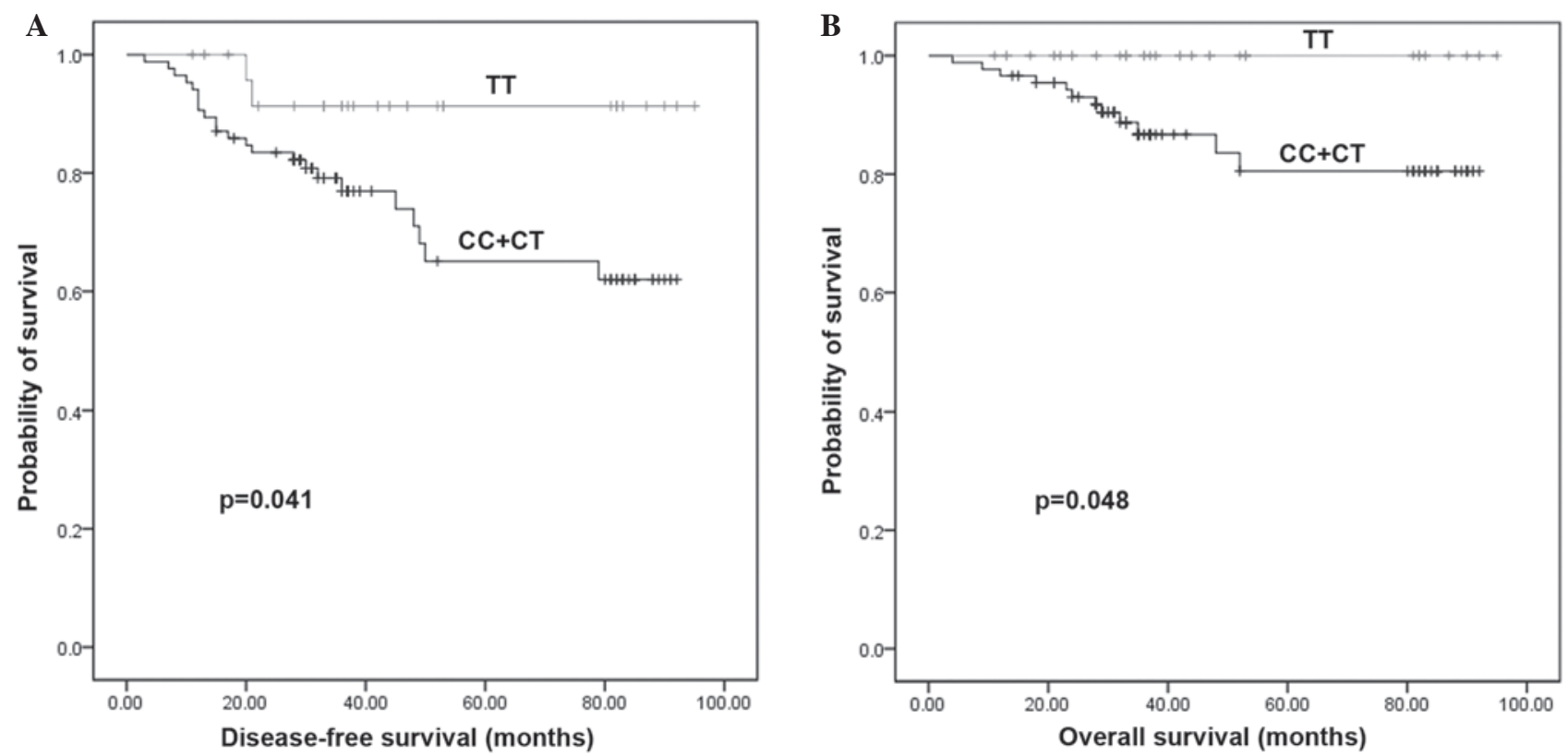

Figure 2. rs621554 polymorphisms were correlated with breast cancer prognosis. (A) The CC+CT genotypes of rs621554 had shorter relapse-free survival $(\mathrm{P}=0.041)$. (B) The $\mathrm{CC}+\mathrm{CT}$ genotypes of rs621554 were associated with shorter overall survival $(\mathrm{P}=0.048)$.

test. The genotype and allele frequencies were tested using Hardy-Weinburg equilibrium (HWE). $\chi^{2}$ test was performed to confirm the representativeness of each group. $\mathrm{P}>0.05$ was considered as non-deviation from HWE. The $\chi^{2}$ result was 3.06 for the patient group and 0.43 for control group, respectively $(\mathrm{P}>0.05)$.

Table I shows a significantly different distribution of rs621554 polymorphism in the patient and control groups. A higher percentage of $\mathrm{CT}$ or $\mathrm{CC}$ genotypes were observed in the patient group compared with the control group (CT: 83.33 vs. $75.45 \%$; CC: 81.12 vs. $67.07 \%$, respectively). Logistic regression analysis showed significant differences between the two groups $(\mathrm{P}=0.032$ and $\mathrm{P}=0.001$, respectively). Moreover, the $\mathrm{CT}$ and $\mathrm{CC}$ genotypes were associated with a higher risk of breast cancer [Odds ratio (OR), 1.627; 95\% confidence interval (CI), 1.041-2.541; and OR, 2.109; 95\% CI, 1.328-3.348, respectively]. Based on the above, our study indicated that the $\mathrm{C}$ allele may be a risk factor for breast cancer susceptibility. Accordingly, the $\mathrm{CT}$ and $\mathrm{CC}$ genotypes were taken together and compared with the TT group.

Association between rs621554 polymorphisms and breast cancer clinicopathological variables. Table II shows the association between the TT and CT+CC genotype and clinicopathological characteristics of breast cancer patients, including age at diagnosis, pathological type, tumor size, histological grade, lymph node status, and biological markers, such as ER, PR and HER2. It was demonstrated that the $\mathrm{CT}$ and $\mathrm{CC}$ genotype were strongly associated with tumor size $(\mathrm{P}=0.035)$, lymph node metastasis $(\mathrm{P}=0.047)$ and $\mathrm{PR}$ status $(\mathrm{P}=0.031)$. No significant association was found between genotype and age at diagnosis, pathological type, histological grade, and status of ER and HER2.

rs621554 polymorphisms are correlated with breast cancer prognosis. The association between rs621554 polymorphisms

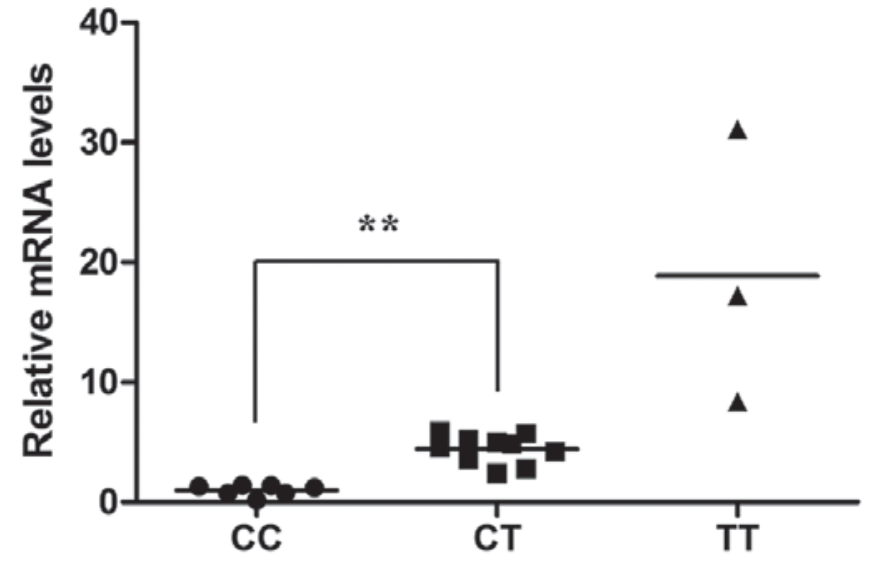

Figure 3. rs621554 polymorphisms are associated with the mRNA level of DLC1 in vivo. The CC genotype resulted in a significantly lower mRNA level of DLC1. No significant differences were identified between CC and TT genotypes, or $\mathrm{CT}$ and TT genotypes. ${ }^{* *} \mathrm{P}<0.01$.

and prognosis was also analyzed using the Kaplan-Meier method. As shown in Fig. 2, compared with patients with the TT genotype, patients with CT and CC genotypes had shorter disease-free survival $(\mathrm{P}=0.041)$ and overall survival $(\mathrm{P}=0.048)$ rates. Additionally, COX hazard logistic regression analysis was performed to demonstrate that rs621554 polymorphisms are not an independent prognostic factor in breast cancer, with $\mathrm{P}=0.192$ for disease-free survival and $\mathrm{P}=0.131$ for overall survival (data not shown).

rs621554 polymorphisms are associated with the mRNA level of DLCl in vivo. The possible correlation between the rs621554 polymorphism and the mRNA level of DLC1 was also investigated. In total, 20 samples of breast cancer tissue were taken for analysis and the result is shown in Fig. 3. The 
relative mRNA level of DLC1 was $1.000 \pm 0.451$ for the CC genotype, $4.452 \pm 1.18$ for the CT genotype, and $18.886 \pm 11.433$ for the TT genotype. Significant differences were identified in the mRNA level between patients with the CC and CT genotype $(\mathrm{P}<0.001)$, and no significance was found between the $\mathrm{CC}$ and TT genotypes $(\mathrm{P}=0.113)$ or the $\mathrm{CT}$ and TT genotypes $(\mathrm{P}=0.160)$.

\section{Discussion}

As with other types of malignancies, genetic and environmental factors are involved in the development of breast cancer. SNPs are the most frequent gene mutation, and certain SNPs have been shown to result in a higher risk of cancer (18). Since the $6 \mathrm{q} 25.1 \mathrm{rs} 2046210$ was first reported by Zheng et al (19) as a novel SNP associated with breast cancer risk, increased attention has been paid to cancer-associated SNPs with the aim of identifying sensitive genetic markers to assess cancer risk and to monitor therapeutic response.

The human DLC1 gene is a tumor suppressor that acts in a Rho-dependent manner, since the protein it encodes is highly homologous to the rat p122-RhoGAP $(20,21)$. It is found to be crucial in regulating the structure of the actin cytoskeleton, and inhibit cell proliferation, migration, invasion and angiogenesis (12-14). It has been shown to be downregulated or absent in a number of different types of cancer (22). According to previous studies, genetic variants widely exist in DLC1 and may influence the antitumor effect of DLC1 $(16,23,24)$. rs621554, located in intron 19, is a synonymous SNP in DLC1, which is associated with HCC (16). Nevertheless, whether the rs621554 polymorphism is associated with breast cancer remains to be determined.

In the present study, the $\mathrm{CT}$ and $\mathrm{CC}$ genotypes (CT+CC genotype) of rs621554 DLC1 were shown to be associated with a significantly higher risk of breast cancer compared with that of the TT genotype. It appears that the T allele of rs621554 DLC1 is protective against breast cancer. Since clinocopathological characteristics are vital in evaluating cancer prognosis their correlation with the rs621554 polymorphism was investigated, and it was demonstrated that the CT+CC genotype was associated with larger tumor size, lymph node metastasis and negative PR status, but was not associated with ER or HER2 status. This may due to the limited number of samples, which suggests that a larger number of cases is required in a further study. In addition, it was demonstrated that the CT+CC genotype was a prognostic indicator for disease-free survival and overall survival in breast cancer, but a COX hazard regression analysis indicated that, with the limited sample size, CT+CC genotype was not shown to be an independent indicator. Despite this, however, a conclusion can be made that the $\mathrm{CT}+\mathrm{CC}$ genotype is associated with breast cancer susceptibility, development and prognosis.

Next, in order to determine the possible correlation between the rs621554 polymorphism and DLC1 expression, DLC1 mRNA expression was detected. It was demonstrated that it is higher in patients with the CT genotype compared with the CC genotype, suggesting the protective effect of the $\mathrm{T}$ allele. However, the DLC1 expression level in patients with the TT genotype is not significantly different compared with that in CT and CC group. This phenomenon may due to small number patients with the TT genotype (only 3 samples) and large standard error. It was hypothesized that the change in the mRNA level of DLC1 due to the SNP may be responsible for the development and progression of breast cancer. The specific mechanism underlying this effect may involve alterations in the binding site of transcriptional factors (25), since rs621554 polymorphism is located in the intron of DLC1, where a variety of cis elements are involved. However, further investigation is required to confirm this.

In conclusion, the results demonstrated that individuals with the CT and CC genotype of the DLC1 rs621554 polymorphism are more susceptible to breast cancer compared with those with the TT genotype and the two genotypes together are associated with larger tumor size, lymph node metastasis and negative PR. In addition, the CT or CC genotype may be associated with a poor prognosis. Further research is required to determine the underlying molecular mechanisms with larger patient cohorts to further confirm rs621554 polymorphism as a clinical biomarker for early diagnosis and prognosis of breast cancer.

\section{Acknowledgements}

This study was supported by the National Natural Science Foundation of China (grant nos. 81272903 and 81172529); the Shandong Science and Technology Development Plan (grant no. 2013GRC31801) and the Special Support Plan for National High Level Talents (Ten Thousand Talents Program) to Dr Qifeng Yang.

\section{References}

1. Basu S, Nachat-Kappes R, Caldefie-Chézet F and Vasson MP: Eicosanoids and adipokines in breast cancer: from molecular mechanisms to clinical considerations. Antioxid Redox Signal 18: 323-360, 2013.

2. Bray F, Jemal A, Grey N, Ferlay J and Forman D: Global cancer transitions according to the Human Development Index (2008-2030): a population-based study. Lancet Oncol 13: 790-801, 2012.

3. Børresen AL: Role of genetic factors in breast cancer susceptibility. Acta Oncol 31: 151-155, 1992.

4. Bishop DT: The involvement of genetic factors in susceptibility to breast cancer. Eur J Cancer Prev 2 (Suppl 3): 125-130, 1993.

5. Mavaddat N, Antoniou AC, Easton DF and Garcia-Closas M: Genetic susceptibility to breast cancer. Molr Oncol 4: 174-191, 2010.

6. Nickels S, Truong T, Hein R, et al: Evidence of gene-environment interactions between common breast cancer susceptibility loci and established environmental risk factors. PLoS Genet 9: e1003284, 2013.

7. Czerska K, Nawara M and Bal J: I. Single nucleotide polymorphism in human genetic analyses. Med Wieku Rozwoj 7: 531-546, 2003 (In Polish).

8. Erichsen HC and Chanock SJ: SNPs in cancer research and treatment. Br J Cancer 90: 747-751, 2004.

9. Zhang N, Li X, Tao K, et al: BCL-2 (-938C > A) polymorphism is associated with breast cancer susceptibility. BMC Med Genet 12: 48, 2011.

10. Romanowicz-Makowska H, Bryś M, Forma E, et al: Single nucleotide polymorphism (SNP) Thr241Met in the XRCC3 gene and breast cancer risk in Polish women. Pol J Pathol 63: 121-125, 2012.

11. Popescu NC and Goodison S: Deleted in liver cancer-1 (DLC1): an emerging metastasis suppressor gene. Mol Diagn Ther 18: 293-302, 2014.

12. Yamaga M, Kawai K, Kiyota M, Homma Y and Yagisawa H: Recruitment and activation of phospholipase C (PLC)-delta1 in lipid rafts by muscarinic stimulation of PC12 cells: contribution of p122RhoGAP/DLC1, a tumor-suppressing PLCdelta1 binding protein. Adv Enzyme Regul 48: 41-54, 2008. 
13. Shih YP, Liao YC, Lin Y and Lo SH: DLC1 negatively regulates angiogenesis in a paracrine fashion. Cancer Res 70: 8270-8275, 2010.

14. Zhou X, Thorgeirsson SS and Popescu NC: Restoration of DLC-1 gene expression induces apoptosis and inhibits both cell growth and tumorigenicity in human hepatocellular carcinoma cells. Oncogene 23: 1308-1313, 2004.

15. Zheng SL, Mychaleckyj JC, Hawkins GA, et al: Evaluation of DLC1 as a prostate cancer susceptibility gene: mutation screen and association study. Mutat Res 528: 45-53, 2003.

16. Dong X, Zhou G, Zhai Y, et al: Association of DLC1 gene polymorphism with susceptibility to hepatocellular carcinoma in Chinese hepatitis B virus carriers. Cancer Epidemiology 33: 265-270, 2009.

17. Suzuki Y, Saito R, Zaraket H, Dapat C, Caperig-Dapat I and Suzuki H: Rapid and specific detection of amantadine-resistant influenza A viruses with a Ser31Asn mutation by the cycling probe method. J Clin Microbiol 48: 57-63, 2010.

18. Temin HM: Evolution of cancer genes as a mutation-driven process. Cancer Res 48: 1697-1701, 1988

19. Zheng W, Long J, Gao YT, et al: Genome-wide association study identifies a new breast cancer susceptibility locus at 6q25.1. Nat Genet 41: 324-328, 2009.
20. Yuan BZ, Miller MJ, Keck CL, Zimonjic DB, Thorgeirsson SS and Popescu NC: Cloning, characterization, and chromosomal localization of a gene frequently deleted in human liver cancer (DLC-1) homologous to rat RhoGAP. Cancer Res 58: 2196-2199, 1998.

21. Zhou X, Zimonjic DB, Park SW, Yang XY, Durkin ME and Popescu NC: DLC1 suppresses distant dissemination of human hepatocellular carcinoma cells in nude mice through reduction of RhoA GTPase activity, actin cytoskeletal disruption and down-regulation of genes involved in metastasis. Int J Oncol 32: 1285-1291, 2008

22. Ullmannova V and Popescu NC: Expression profile of the tumor suppressor genes DLC-1 and DLC-2 in solid tumors. Int J Oncol 29: 1127-1132, 2006.

23. Xie CR, Sun HG, Sun Y, et al: Significance of genetic variants in DLC1 and their association with hepatocellular carcinoma. Mol Med Rep 12: 4203-4209, 2015.

24. Dai X, Li L, Liu X, Hu W, Yang Y and Bai Z: Cooperation of DLC1 and CDK6 affects breast cancer clinical outcome. G3 (Bethesda) 5: 81-91, 2014

25. Lee PH and Shatkay H: F-SNP: computationally predicted functional SNPs for disease association studies. Nucleic Acids Res 36: D820-D824, 2008. 